\title{
Replication of Finn \& Hudson Kam (2008) The curse of knowledge: First language knowledge impairs adult learners' use of novel statistics for word segmentation, Exp. 1
}

\author{
Caitlin R. Garcia, Kendall D. van Horne and Joshua K. Hartshorne \\ Department of Psychology, Boston College \\ Contact joshua.hartshorne@bc.edu
}

\begin{abstract}
We replicated Exp. 1 of Finn \& Hudson Kam (2008) The curse of knowledge: First language knowledge impairs adult learners' use of novel statistics for word segmentation, Cognition, $108,477-499$, as part of a multi-year effort to replicate every adult statistical word segmentation study. Unlike the original study, we do not find clear evidence that English-speaking adults fail to successfully segment words that violate the phonotactic constraints of English.
\end{abstract}

Keywords: statistical learning, unsupervised learning, language acquisition, replication

In this paper, we report a replication of Experiment 1 from Finn \& Hudson Kam (2008), henceforth FHK08. This experiment was part of a larger project to systematically replicate as many experiments involving statistical word learning in adults as possible.

\section{Method}

\section{Subjects}

100 subjects were recruited through Amazon Mechanical Turk and paid for their participation. 29 were excluded for missing more than one catch trial, leaving 37 in the control condition (Ages 23-61, M=33) and 34 in the experimental condition (Ages 24-63, M=37). All but one were native English speakers, and all reported normal hearing.

\section{Known differences with original.}

- Our study had nearly double the number of subjects (40 vs. 71) and thus considerably more power and precision.

- Subjects in our study were likely older than the undergraduate subjects in the original.

- Subjects in our study were compensated monetarily rather than with course credit.

\section{Materials}

Training. The stimuli cannot be easily recreated: The description in the original paper uses an obsolete phonetic alphabet for which we were unable to locate a key. The authors graciously provided us with the original stimuli. These stimuli are now available as part of the repository associated with the present project, with permission of the authors (osf.io/fbzkt).
Experimental and control stimuli both consisted of eight two syllable words (CCVCV), each beginning with a consonant cluster. For the experimental stimuli, these $\mathrm{CC}$ onsets violate the word-initial phonotactic rules of English. In the control stimuli, CC onsets are licit. FHK08 report that the stimuli were generated with the text-to-speech program SoftVoice. The synthesizer produced syllables with a monotonic F0 (fundamental frequency) of $83.62 \mathrm{~Hz}$. All vowels were matched for length, and there were no co-articulation effects.

During training, words were presented quasi-randomly with no pauses and no immediate repetitions. FHK08 report transitional probabilities of 1.0 for syllable transitions that are word-internal and .143 at word boundaries. They further report that phoneme transitional probabilities (PTPs) within words were higher than those across word boundaries, with word-internal PTPs, ranging from .25 to 1.0 and PTPs across the word boundaries ranging from .035 to .143 . Thus, there was no overlap in the within-word and across-word PTPs.

Training lasted $17 \mathrm{~min} 59 \mathrm{sec}$. While FHK08 report that each word occurred 560 times, we suspect this is a type-o, since it would suggest 8.3 syllables/sec. The speech rate in the training files provided is much closer to 1 syllable/sec, which would be consistent with each word occurring 70 times for a total of 560 words in the training. However, we did not confirm this with an exact count of the words.

Test. After exposure, participants were given a forcedchoice test between a trained word and either a non-word (consisting of two syllables from the language but a transitional probability of 0 ) and a split-cluster (a trained word minus the first consonant and with another trained word's initial consonant at the end, resulting in a CVCVC structure). In essence, the researchers constructed the split-cluster words by shifting one phoneme to the right in the exposure stimuli. Note that in the experimental condition, this results in a foil 
that is a licit English word, pitted against a target which is not. The split-cluster foils measured the accuracy of parsing the consonant clusters in defiance of English phonotactics, while the non-word foils served as a (high) baseline.

There were 8 of each type of test item (word vs. nonword, word vs. split-cluster word), yielding 16 test items in total for each language. The two items in a pair were presented one after another with a 1 second pause in between. There was a $500 \mathrm{~ms}$ pause in between pairs during which participants were expected to answer. The order of test trials was randomized for each subject.

Upon inspection, two of the nonwords (one in the control condition and one in the experimental condition) did not have an audible vowel in the first syllable, resulting in a CCCV structure. ${ }^{1}$ In principle, this should have made it easy for the subjects to reject the nonword, though, in fact, accuracy was high for one (0.89) but not the other (0.53). Below, we report analyses both with and without these items.

In addition to these trials, we included eight catch trials that pitted a trained word against a nonword that included phonemes that never appeared in the training. These nonwords were created using MBROLA (Dutoit et al., 1996). Due to experimenter error, one of the catch trials in the experimental condition did not have a correct answer, and so was excluded. The catch trials were always the last eight items in the test, and the order of the catch trials was randomized for each subject.

\section{Known differences with original.}

- FHK08 had a $3000 \mathrm{~ms}$ pause after test trials, whereas we used $500 \mathrm{~ms}$. This was done to prevent subjects from thinking the web page was broken and refreshing.

- FHK08 did not include catch trials. Note that since our catch trials follow the critical trials, they cannot affect performance on the critical trials.

- FHK08 assigned subjects to one of two orders of test trials, each of which was the reverse of the other. We randomized ours independently for each subject.

- In FHK08, subjects responded to the test with paper and pencil. Our test was computerized.

\section{Procedure}

Participants were informed that they would be listening to a new language and were instructed to listen to it as best as they could, but were told to be careful not to overthink or ignore what they were listening to. There were two separate listening blocks to allow subjects to take a break between the two sets of training. After this exposure, they were told they would be tested to see what they learned about the language. They were encouraged to draw a picture during the exposure phase. At the end of the experiment, they were required to describe their drawing. Six of the subjects reported forgetting to draw anything.

After exposure, participants were given the forced-choice test described above. They were instructed to listen to pairs of possible words and were asked to choose which word was a better example of the language. Participants indicated responses using two keys on their keyboard, pressing ' 1 ' if the first item in the pair sounded closer to the language and ' 2 ' if the second one sounded closer to the language. After the test, participants completed an additional survey consisting of questions regarding their demographic and language backgrounds.

\section{Known differences with original.}

- FHK08 do not report how many of their subjects drew pictures during the training. However, since they provided subjects with drawing materials, it is reasonable to assume that more did so in their study than in ours.

- Our subjects were tested online, whereas FHK08's were tested in the laboratory.

- Subjects in our experiment used their own equipment, increasing variability across subjects. It is not clear what equipment was used by FHK08, but presumably it was more stable subject-to-subject.

\section{Results}

All data and code are available through the Open Science Framework at osf.io/fbzkt.

Original Analyses. Performance on the non-word items was above chance in both the control condition $(\mathrm{M}=71 \%$, $\left.\mathrm{SE}=3 \%, \mathrm{t}(36)=7.4, \mathrm{p}=9.6^{*} 10^{-9}\right)$ and in the experimental condition $\left(\mathrm{M}=62 \%, \mathrm{SE}=3 \%, \mathrm{t}(33)=4.6, \mathrm{p}=6.8 * 10^{-5}\right)$. Performance was significantly better in the control condition $(\mathrm{t}(69)=2.4, \mathrm{p}=.02)$. This is inconsistent with the results from Finn et al. (2008), in which the groups did not differ significantly.

Performance on the split-cluster test was significantly above chance in the control condition $(\mathrm{M}=62 \%, \mathrm{SE}=3 \%$, $\left.\mathrm{t}(36)=4.8, \mathrm{p}=2.9 * 10^{-5}\right)$, consistent with FHK08. While it was only marginally above chance in the experimental condition $(\mathrm{M}=56 \%, \mathrm{SE}=3 \%, \mathrm{t}(33)=1.9, \mathrm{p}=.061)$, the difference between conditions did not reach significance $(\mathrm{t}(69)=1.5$, $\mathrm{p}=.15$ ). This contrasts with FHK08, who found a significant difference between the two conditions. Whether we replicated their finding of chance performance in the experimental condition depends on how one interprets $p=.06$. Certainly, it is at best a weak confirmation.

Removing the two problematic test items mentioned above had minimal effect on the pattern of results. The difference between conditions when tested on non-word items

\footnotetext{
${ }^{1}$ In the repository, these are expernon5 and controlnon 4
} 
was no longer significant $(\mathrm{t}(69)=1.3, \mathrm{p}=.19)$. Otherwise, the pattern of significance was the same. Removing the six subjects who forgot to draw during the training likewise had no effect on the pattern of significance. Removing both those subjects and the problematic test items resulted in the same pattern of significance as removing only the two problematic test items. Thus, these analysis decisions have little to no impact on interpretation of the results.

\section{Revised Analyses}

There are two important limitations to the original analyses conducted by FHK08. First, their use of t-tests on accuracy data violates core assumptions of t-tests: that the data are continuous and error is normally distributed. Violation of these assumptions can lead both to false positives and false negatives (Jaeger, 2008). Binomial models are more appropriate (Jaeger, 2008). ${ }^{2}$

Additionally, they treat items as fixed effects. Thus, any conclusions are specific to the stimuli used. No assessment of generalization can be made. Statisticians have suggested two options. The first is to conduct analyses separately over subject averages and over item averages (Clark, 1973). The second option is to use mixed effects models (Baayen et al., 2008).

Given these considerations, we re-analyzed the data used binomial mixed effects models, implemented in $\mathrm{R}$ using the lme4 package, ver. 1.1-12 (Bates et al., 2017). We included fixed effects for condition and foil type (non-word vs. splitcluster) and their interaction. We included random intercepts both for subject and for target/foil pair, plus a random slope of test type (nonword vs. split-cluster) by subject. We set the contrast structure to center the variables, which results in the intercept being the grand mean.

We find a significant intercept, reflecting overall successful learning of the trained words $(\mathrm{B}=.56$, Wald's $\mathrm{z}=5.2$, $\left.\mathrm{p}=2.3^{*} 10^{-7}\right)$. Neither of the main effects nor the interaction were significant ( $\mathrm{ps}>\mathrm{.1})$. Follow-up analyses looking at the each condition and foil-type individually revealed significant intercepts for non-words in the control condition (Wald's $z$ $=4.2, p=.00004)$, split-clusters in the control condition (Wald's $z=2.6, p=.008$ ), non-words in the experimental condition (Wald's $z=2.4, p=.02$ ), but not split-clusters in the experimental condition (Wald's $z=1.1, \mathrm{p}=.28$ ).

The pattern of significance was identical when excluding the two bad items, the subjects who forgot to draw, or both.

\section{Discussion}

The primary theoretically-relevant findings reported by FHK08 was that English-speaking adults failed to successfully segment words that violated the phonotactic constraints of English but could successfully segment phonotacticallylicit words. However, subjects could reject foils that had a between-syllable transition probability of 0 equally well in both conditions.

In contrast, we find that accuracy is above chance, with no evidence of any effect of phonotactics or foil type. This is particularly true of our revised analyses, which take into account variance in the stimuli. The one caveat to this is that the above-chance rejection of split-cluster foils does not rise to the level of significance when considered on its own. This could mean that we do not have the power to detect abovechance performance for that class of stimuli. Alternatively, it may mean that we do not have the power to detect a difference in performance between that class and other classes of stimuli. The overall effect, however, is that we are unable to find clear support of the original finding.

It is certainly possible that these factors have an effect on performance, but that we did not have enough power to detect them. Note that this concern applies even more strongly to FHK08, who had far fewer subjects.

It is also possible that the differences in results reflect a difference in subject population (AMT workers vs. Berkeley undergraduates) or venue (online vs. brick-and-mortar lab). However, typically these concerns would lead us to expect worse performance for the subjects in our replication. The biggest difference between our findings and the original is that we find better performance in the experimental condition on the split-cluster foils than FHK08 did.

Note that while we were not able to confirm that statistical word segmentation is modulated by the factors investigated by FHK08, we do find clear evidence of successful statistical word segmentation.

\section{Materials}

All data, materials, and analysis scripts can be found at osf.io/fbzkt.

\section{References}

Baayen, R. H., Davidson, D. J., \& Bates, D. M. (2008). Mixedeffects modeling with crossed random effects for subjects and items. Journal of Memory and Language, 59(4), 390-412.

Bates, D., Maeschler, M., Bolker, B., Walker, S., Christensen, R. H. B., Singmann, H., .. Green, P. (2017). Linear mixed-effects models using 'eigen' and s4. cran.rproject.org/web/packages/lme4/index.html.

Clark, H. H. (1973). The language-as-fixed-effect fallacy: A critique of language statistics in psychological research. Journal of Verbal Learning and Verbal Behavior, 12(4), 335-359.

Dutoit, T., Pagel, V., Pierret, N., Bataille, F., \& Van der Vrecken, O. (1996). The mbrola project: Towards a set of high quality speech synthesizers free of use for non commercial purposes. In Spoken

\footnotetext{
${ }^{2} \mathrm{An}$ intermediate option is to transform accuracy measures to log-odds, though this method is less accurate than a binomial model.
} 
language, 1996. icslp 96. proceedings., fourth international conference on (Vol. 3, pp. 1393-1396).

Finn, A. S., \& Hudson Kam, C. L. (2008). The curse of knowledge: First language knowledge impairs adult learners' use of novel statistics for word segmentation. Cognition, 108(2), 477-499.
Jaeger, T. F. (2008). Categorical data analysis: Away from ANOVAs (transformation or not) and towards logit mixed models. Journal of Memory and Language, 59(4), 434-446. doi: 10.1016/j.jml.2007.11.007 